\title{
Beziehungsqualitäten in Kita und Schule
}

\author{
Zusammenhänge mit akademischen Kompetenzen, \\ Motivation, sozialer Integration und Wohlbefinden
}

\author{
Bettina Hannover \\ Freie Universität Berlin, Deutschland
}

\begin{abstract}
Zusammenfassung: Die affektive Qualität der Beziehung, die zwischen Kind oder Jugendlichen und der pädagogischen Fachkraft in Kita oder Schule besteht, ist nicht nur für die sozioemotionale und akademische Entwicklung des Lernenden bedeutsam, sondern korrespondiert auch dem Wohlbefinden und Kompetenzerleben der Erziehungs- oder Lehrperson. In diesem Themenheft der Zeitschrift für Pädagogische Psychologie sind Beiträge zusammengestellt, die die vielschichtigen Dimensionen reflektieren, auf denen Beziehungsqualität beschrieben und analysiert werden kann. Mit der Unterschiedlichkeit der behandelten Fragestellungen möchte das Themenheft auch eine Anregung für weitere Forschung zum Thema Beziehungsqualität in Kita und Schule bieten.
\end{abstract}

Schlüsselwörter: Pädagogische Beziehungen, Beziehungsqualität, motivationale und kognitive Entwicklung, Belastungserleben, Wohlbefinden

Relationship qualities in daycare and school. Links with academic competencies, motivation, social integration, and well-being.

Abstract: The relationship that a child or adolescent has with the care provider or teacher can differ significantly in its affective quality. Relationship quality has been shown to be significant not only for the motivational and cognitive development of the learning person, but also for well-being and experience of competence of the care provider or teacher. This special issue of the Zeitschrift für Pädagogische Psychologie includes articles that reflect the multifaceted dimensions on which relationship quality can be described and analyzed. With the diversity of the topics addressed, the special issue also aims to stimulate further research on the topic of relationship quality in daycare centers and schools.

Keywords: Relationships in educational settings, relationship quality, motivational and cognitive development, experience of stress, well-being

Die Qualität der Beziehung zwischen Erziehungsperson oder Lehrkraft auf der einen Seite und dem Kind oder Jugendlichen auf der anderen Seite ist in unterschiedlichen Forschungstraditionen untersucht worden: der Bindungsforschung, der Erziehungsstilforschung, der Führungsstilforschung, der Forschung über Lehrstile, der Forschung zur Selbstbestimmungstheorie sowie innerhalb von mit dem Interpersonalen Zirkumplex verbundenen Ansätzen zur Interpersonal Theory. In diesem Themenheft der Zeitschrift für Pädagogische Psychologie sind Beiträge zusammengestellt, die Beziehungsqualität in der Kita oder Schule in ihren Zusammenhängen zu akademischen, motivationalen, sozialen oder emotionalen Ergebnissen für das Kind, den Jugendlichen oder die pädagogische Fachkraft untersuchen. Dabei liegt der Fokus auf der affektiven Qualität der Beziehung- d.h. auf Gefühlen und emotional relevanten Einschätzungen, die die Kinder oder Jugendlichen und die pädagogische Fachkraft in Bezug aufeinander haben.
Die Unterschiedlichkeit der Studien zeigt die Komplexität des Themas auf. Eine Dimension, auf der sich die Beiträge des Themenheftes unterscheiden lassen, stellen die Inhalte der Beziehungsqualität dar. Ilka Wolter und Bettina Hannover beobachten Bindungsqualität, die definiert ist über die Gewährung emotionaler Unterstützung bei gleichzeitiger Unterstützung des Explorationsverhaltens des Kindes. Bettina Hannover, Karoline Koeppen und Madeleine Kreutzmann lassen Lehrkräfte ihr Verhalten gegenüber einem Kind in einem Zirkumplex beschreiben, der über die beiden orthogonalen Dimensionen Communion (Wärme, Herzlichkeit usw.) und Agency (Lenkung, Kontrolle usw.) aufgespannt wird. Jasmin Decristan, Mareike Kunter und Benjamin Fauth sowie Frances Hoferichter und Diana Raufelder bitten Schülerinnen und Schüler anzugeben, wie stark sie sich von ihrer Mathematiklehrkraft bzw. ihren Lehrkräften sozio-emotional und fachlich-inhaltlich in ihrer Lernentwicklung unterstützt fühlen. Und Julia Roza, Anne Frenzel und Robert Klassen 
lassen Lehrkräfte beschreiben, wie sie die Beziehung zu ihrer Klasse affektiv erleben.

Manche der Beiträge zum Themenheft fokussieren auf Unterschiede in der Beziehungsqualität in Abhängigkeit von Merkmalen der Lernenden (Noten, Erstsprache, sonderpädagogischer Förderbedarf und Geschlecht bei Hannover et al.; Geschlecht bei Wolter \& Hannover) und andere auf die Identifikation von Zusammenhängen zwischen Beziehungsqualität und Ergebnisvariablen (mathematikbezogene Kompetenz und Motivation der Kinder bei Wolter \& Hannover; soziales Integrationserleben der Schülerinnen und Schüler bei Decristan et al.; Stresserleben und schulisches Erschöpfungserleben der Schülerinnen und Schüler bei Hoferichter \& Raufelder; berufs- und unterrichtsbezogene Emotionen, Burnout und Selbstwirksamkeitserleben der Lehrkraft bei Roza et al.), die sich ebenfalls in Abhängigkeit von Merkmalen der Personen unterscheiden können (wie bei Decristan et al. in Abhängigkeit des Fähigkeitsselbstkonzepts und der Leistungsängstlichkeit der Schülerinnen und Schüler).

Auch können die Beiträge danach differenziert werden, wer das Subjekt der Untersuchung ist, nämlich die jeweilige Beziehung beschreibt oder beobachtet. Hannover et al. und Roza et al. erheben Einschätzungen der Lehrkraft. Decristan et al. und Hoferichter und Raufelder arbeiten hingegen mit den Urteilen der Schülerinnen und Schüler. Und bei Wolter und Hannover beobachten Externe die Bindungsqualität. Weiter unterscheidet sich nun, wer oder was das Objekt der Beurteilung oder Beobachtung ist: wird eine dyadische Beziehung beschrieben (wie bei Wolter \& Hannover, wo jeweils eine Erzieherin-Kind-Dyade beobachtet wird oder bei Decristan et al., wo jeder Lernende über seine Beziehung zur Mathematiklehrkraft berichtet oder bei Hannover et al., wo die Lehrkraft ihr Verhalten gegenüber einem Grundschulkind darstellt), oder wird die Beziehung zu einer Gruppe von Personen beschrieben (wie bei Hoferichter \& Raufelder, wo jeder Lernende über die Beziehung berichtet, die die Lehrkräfte (Plural) zu ihm haben oder bei Roza et al., wo die Lehrkraft ihre Beziehung zu ihrer Klasse einschätzt). In einer sehr informativen Zusammenstellung benennen Roza et al. in ihrem Beitrag Messinstrumente, die zur Erfassung von Beziehungsqualitäten für einige der verschiedenen möglichen Kombinationen von Subjekt und Objekt der Beziehung vorliegen (siehe dort Tabelle 1).

\section{Die Beiträge im Einzelnen}

Im Themenheft werden die Beiträge in der Abfolge der Alters- oder Entwicklungsphase der Personen dargestellt, die im Fokus der jeweiligen Untersuchung stehen. Der erste Beitrag bezieht sich auf Kinder in der Kindertagesstätte
(Wolter \& Hannover), der zweite auf Kinder in der Grundschule (Hannover et al.), der dritte (Decristan et al.) und vierte Beitrag (Hoferichter \& Raufelder) auf Schülerinnen und Schüler in der Sekundarstufe und der fünfte und letzte Beitrag auf die Situation der beruflich tätigen Lehrkraft (Roza et al.).

Wolter und Hannover untersuchen die Bindungsqualität in der dyadischen Beziehung zwischen Erzieherin und Kind sowie die Aktivitätsangebote, die die Erzieherin macht. Als abhängige Variablen werden mathematikbezogene Kompetenzen und Motivation des Kindes untersucht. Die Ergebnisse zeigen, dass Mädchen in signifikant qualitätsvollere Bindungsbeziehungen mit ihren Erzieherinnen eingebunden waren als Jungen. Unabhängig von der Beziehungsqualität stieg mit der Häufigkeit des Angebots mathematik- und naturwissenschaftsbezogener Aktivitäten die mathematikbezogene Motivation der Mädchen, während sie für die Motivation der Jungen keine Rolle spielte.

Hannover, Koeppen und Kreutzmann lassen Lehrkräfte ihr Verhalten in dyadischen Beziehungen zu Grundschulkindern beschreiben. Die Autorinnen prüfen, ob Lehrkräfte das Ausmaß, indem sie das Verhalten eines Kindes steuern und überwachen (Agency), an den durch Noten gemessenen Kompetenzen des jeweiligen Kindes ausrichten und ob Lehrkräfte sich gegenüber verschiedenen Gruppen von Kindern unterschiedlich stark agentisch verhalten. Erwartungsgemäß passten die Lehrkräfte ihr Verhalten gegenüber einem Kind an seine Kompetenzen an. Wurden die Selbstangaben der Lehrkräfte getrennt danach betrachtet, auf welche Gruppen von Kindern sie sich bezogen, wurden jedoch auch Unterschiede sichtbar, die nicht mit den Kompetenzen der Kinder erklärt werden konnten. So zeigten die Lehrkräfte z.B. gegenüber Jungen häufiger Verhaltensweisen, die durch hohe Agency und gleichzeitig geringe Wärme charakterisiert sind.

Decristan, Kunter und Fauth untersuchen die Bedeutung sozio-emotionaler Unterstützung und fachlich-inhaltlicher Unterstützung durch die Mathematik-Lehrkraft für die von den Schülerinnen und Schülern erlebte soziale Integration im Mathematikunterricht und differenzielle Zusammenhänge in Abhängigkeit der Lernvoraussetzungen der Schülerinnen und Schüler, nämlich ihres Fähigkeitsselbstkonzepts und ihrer Leistungsängstlichkeit in der Mathematik. Die Ergebnisse zeigen, dass Schülerinnen und Schüler mit niedrigem Mathematik-Fähigkeitsselbstkonzept dann, wenn ihre Lehrkraft eine starke fachlich-inhaltliche Unterstützung bot, sich nicht weniger sozial integriert fühlten als ihre Mitschüler und Mitschülerinnen ohne ein solches ungünstiges Fähigkeitsselbstkonzept. Das geringere Integrationserleben von Schülerinnen und Schülern mit hoher Mathematik-Leistungsängstlichkeit wurde sowohl durch eine starke sozio-emotionale als 
auch eine starke fachlich-inhaltliche Unterstützung der Lehrkraft moderiert. Die Ergebnisse veranschaulichen, dass eine gute Beziehungsqualität zwischen Lehrkraft und Lernenden den Einfluss nachteiliger individueller Merkmale des Lernenden kompensieren kann.

Ähnlich geht es im Beitrag von Hoferichter und Raufelder darum, ob das Erleben von Unterstützung (sozioemotionale Unterstützung, akademische Unterstützung, Lob und Anerkennung) durch die Lehrkräfte Stress- und Erschöpfungserleben bei Sekundarschülerinnen und -schülern abfedern kann. Schülerinnen und Schüler, die sich unterstützt fühlten, berichteten weniger allgemeinen Stress und schulisches Erschöpfungserleben. Weiter zeigten sich Moderationseffekte der auf die akademische Lernunterstützung bezogenen Variablen auf das Stressund Erschöpfungserleben über ein Schuljahr (Längsschnitt) hinweg: unter Kontrolle von Geschlecht, Schulform und Noten trug wahrgenommene Lernunterstützung durch die Lehrkräfte dazu bei, dass die schulische Erschöpfung des Schülers oder der Schülerin über das Schuljahr abnahm.

Roza, Frenzel und Klassen haben in zwei Studien ein neues Messinstrument entwickelt und validiert, mit dem die affektive Beziehung der Lehrkraft zu ihrer Klasse aus Sicht der Lehrkraft erfasst werden kann (teacher-class relationship). Roza et al. können zeigen, dass eine positive Beziehung der Lehrkraft zu ihrer Klasse in jeweils erwarteter Richtung mit den auf diese Klasse bezogenen Emotionen beim Unterrichten (teaching emotions), mit der Selbstwirksamkeit als Lehrkraft, mit Burnout und Emotionsarbeit (emotional labor) zusammenhing.

In der Zusammenschau veranschaulichen die Beiträge des Themenheftes unterschiedliche Aspekte oder Dimen- sionen, die eine qualitätsvolle Beziehung zwischen Erziehungs- oder Lehrperson und Kind oder Jugendlichem ausmachen. Sie zeigen, dass sich Beziehungsqualitäten in Abhängigkeit von Merkmalen der Lernenden unterscheiden können, dass hohe Beziehungsqualität sowohl für die Lernenden als auch für die pädagogische Fachkraft mit günstigen Ergebnissen sowie der Abschwächung von Risiken einhergeht und dass der Zusammenhang zwischen Beziehungsqualität und Ergebnisvariablen wiederum durch Merkmale der Lernenden moderiert sein kann.

Betrachtet man die oben dargestellten Dimensionen, auf denen Studien zur Beziehungsqualität in Kita und Schule klassifiziert werden können, so wird deutlich, dass mit den in diesem Themenheft zusammengestellten Beiträgen nur einige wenige der möglichen Kombinationen von Ausprägungen auf diesen Dimensionen untersucht worden sind. Wir hoffen, mit dem Themenheft wird weitere Forschung zur Beziehungsqualität in Kita und Schule angeregt. Insbesondere wird sich zukünftige Forschung auch der Frage widmen müssen, wie pädagogisches Personal noch stärker für die positiven Effekte hoher Beziehungsqualität und die negativen Effekte g eringer B eziehungsqualität s ensibilisiert und dabei unterstützt werden kann - im Interesse der Lernenden und im eigenen Interesse - hochwertige Beziehungsangebote machen zu können.

Onlineveröffentlichung: 23.12.2021

\section{Bettina Hannover}

Freie Universität Berlin

Fachbereich Erziehungswissenschaft und Psychologie

Habelschwerdter Allee 45

14195 Berlin

bettina.hannover@fu-berlin.de 